\title{
Correlations of Online Social Network Size With Well-Being and Distress: A Meta-Analysis
}

\author{
Chiungjung Huang \\ National Changhua University of Education, Changhua, Taiwan
}

\begin{abstract}
This meta-analysis examines the correlations of the number of social network site (SNS) friends with well-being and distress, based on 90 articles consisting of 98 independent samples on correlations of online social network size (OSNS) with happiness, life satisfaction, self-esteem, anxiety, depression, combined anxiety and depression, loneliness, social anxiety, social loneliness, well-being and distress. The correlations between OSNS and well-being indicators are positively weak (from .06 to .15), whereas those for distress indicators are inconclusive (from -.19 to .08). Studies recording the OSNS based on the participant profile have larger mean effect sizes for well-being (.21) and self-esteem (.31) than those based on self-reporting (.06 and .05, respectively). The correlation between OSNS and self-esteem is stronger in samples with a smaller mean network size.
\end{abstract}

Keywords: Social network; well-being; distress; meta-analysis

\section{Introduction}

The traditional definition of mental health is the absence of mental illness (Westerhof \& Keyes, 2010). This traditional view is a one-dimensional model focused on psychopathology, such as depression and anxiety. The dual-factor model (Suldo \& Shaffer, 2008) or two-continuum model (Westerhof \& Keyes, 2010) proposes two related dimensions for the presence or absence of mental health and of mental disorders. Thus, the first continuum focuses on well-being, and the second focuses on distress. Based on the two-continuum model, Keyes (2007) suggested that both positive and negative mental health indicators should be measured for a complete assessment of mental health.

Two major philosophical models are proposed to conceptualize well-being. From the hedonic perspective, wellbeing is defined as happiness, comprising satisfaction with life and with other important domains, positive affect and low negative affect (Deci \& Ryan, 2008; Diener, 2000). From the eudaimonic perspective, well-being is defined as good functioning and fulfillment of life. Ryff (1989) defined the six components of well-being as self-acceptance, positive relations with others, autonomy, environmental mastery, purpose in life and personal growth. Hence, the hedonic standpoint views well-being as an outcome of happiness, whereas the eudaimonic approach considers well-being as a process of actualizing an individual's potential (Deci \& Ryan, 2008).

Two theories of social relationships have been developed for predicting the correlations of social network size with well-being and distress. The social convoy model (Antonucci et al., 2014; Levitt et al., 1993) suggests that family and friends served as a protective layer that can provide social support to help an individual to cope with life stress. Social convoys vary in their closeness. The closest social partners are considered to be in the inner circle, followed by those in the middle and peripheral circles. The relationships with peripheral-circle members are 
less stable than those for inner- and middle-circle members. The number of contacts in the peripheral circle decreases in adulthood, whereas that in the inner circle is maintained throughout a person's life span (Wrzus et al., 2013). The assessment of social convoys is both objective and subjective. The objective measure of social relations refers to the structure, and the subjective measure is the perceived quality of social support. The structure of social convoys, such as total network size, is related to health (Fiori et al., 2007). Furthermore, a sufficient number of social ties is essential to maintain the quality of social relations (Antonucci et al., 2014). Thus, social network size is expected to be an important determinant of well-being and distress. The socioemotional selectivity theory (Carstensen, 1995; Carstensen et al., 1999) posits that the social network size depends on social goals that change with various life stages. Goals can be classified into present- and future-oriented. Futureoriented goals, such as making new friends and acquisition of knowledge, are valuable in the future. The presentoriented goal is related to emotional regulation. Old adults do not perceive an unlimited future life span, and they tend to adopt present-oriented goals. To maximize emotional meaning, old adults decrease the network size and increase social interactions with close ties (Löckenhoff \& Carstensen, 2004). In other words, the maintenance of high emotional closeness with a relatively small number of social partners is related to high emotional well-being for old adults. In contrast, adolescents and young adults perceive an unlimited future life span, and tend to adopt future-oriented goals, such as information acquisition. Adolescents and young adults are likely to maintain large social networks in order to facilitate news-seeking. Thus, increasing social network size facilitates well-being for youths and young adults.

Because social networks can provide social support, social influence, social engagement, face-to-face contacts and access to resources, Smith and Christakis (2008) suggested that such networks are an important determinant of health. Empirical evidence indicates that offline social network size is correlated with well-being and distress. For example, Domènech-Abella et al. (2017) sampled 3,535 Spanish adults older than 50 years, and found that individuals with a smaller social network were more likely to feel lonely. An international study by Litwin and colleagues (2015), who sampled 28,756 adults older than 65 years from Austria, Belgium, Czech Republic, Denmark, Estonia, France, Germany, Hungary, Italy, Netherlands, Poland, Portugal, Slovenia, Spain, Sweden, and Switzerland, found a negative correlation between social network size and depression $(r=-.03)$. Cross-cultural evidence was found in a Chinese population: Chan and Lee (2006) selected adults older than 60 years from Beijing and Hong Kong, and found that individuals with a larger social network felt happier than those with a smaller social network $(r=.18)$. Moreover, the presence of a positive correlation between social network size and subjective well-being $(r=.39)$ was also supported by Wang (2016). However, most of these studies have focused on adults in later life, with relatively few studies examining this correlation among adolescents or young adults.

The increasingly widespread and intensive use of social network sites (SNSS) has changed how individuals make friends and connect with others, potentially enlarging their social networks. Especially for shy individuals, SNSs provide a non-stressful platform to connect with others (L. R. Baker \& Oswald, 2010). However, as the number of friends increases, the amount of time devoted to each friend decreases. An increase in social network size may occur at the expense of the quality of social relationships, reducing support from social partners (Manago et al., 2012). A larger social network may be correlated with higher distress and lower well-being.

\section{Research on the Correlations of OSNS With Well-Being and Distress}

Empirical findings about the direction of the correlation between OSNS and well-being have been inconsistent. For example, Yang and Brown (2016) sampled 218 freshmen, and found a positive correlation between the number of Facebook friends and self-esteem, with $r=.22$. In contrast, Kalpidou and colleagues (2011) found negative correlations when they sampled 35 first-year and 35 upper-class undergraduate students, with $r=-.29$ and $r=-.04$, respectively. Further, the magnitude of the correlation between OSNS and well-being has also varied. Weak correlations were found by Utz and colleagues (2012), who used two samples of Dutch users to examine the correlation between the number of SNS friends and self-esteem, and found $r=0$ and $r=.04$. In contrast, Brailovskaia and Bierhoff (2020) found moderate-to-strong correlations between the number of Facebook friends and self-esteem when they sampled 138 late millennials and 116 early millennials in Germany, with $r=.37$ and $r$ $=.43$, respectively. Similarly, the direction and magnitude of the correlation between OSNS and distress have also varied. Specifically, the correlation between OSNS and loneliness has varied from weakly positive (Bourke, 2013) to moderately negative (Arianna, 2014). 


\section{Source Descriptors}

Findings about the directions and magnitudes of the correlations of OSNS with well-being and distress are inconclusive, and moderator effects are possible. Potential moderators were source descriptors (Lipsey \& Wilson, 2001), such as study country and publication status. To study the country effect, Lönnqvist and Deters (2016) sampled 153 US undergraduate students and 187 German university students and found a noticeable difference between the two samples. The correlations between the number of Facebook friends and life satisfaction were $r$ $=.29$ and $r=.15$, respectively. Regarding the effect of publication status, Ferguson and Heene (2012) suggested that studies producing significant findings were more likely to be published than those producing non-significant findings. Publication bias refers to "the overrepresentation in the literature of studies with significant outcomes compared with studies with null results" (Augusteijn et al., 2019, p. 117) due to the publication decision and the intention of researchers to submit a manuscript.

\section{Moderators Related to Research Context}

Another category of moderators is related to research context. For example, the mean number of friends in the sample may moderate the correlations of OSNS with well-being and distress. While SNSs provide platforms to establish large social networks, time constraints may affect the frequency of communication with friends. As the social network size increases, the time available to maintain each friendship decreases, and so the individual relationships may weaken. Individuals with a small social network may spend more time with each friend, resulting in a high emotional intensity (Pollet et al., 2011; Roberts \& Dunbar, 2011). Hence, the correlations of social network size with well-being and distress may be weaker for studies involving larger social networks. Other context variables included study SNS and how the information about the OSNS was obtained. Most studies measured Facebook friends, while some studies assessed global number of SNS friends or Instagram followers. Further, many studies obtained the number of SNS friends from self-report measures, whereas some others recorded the number of SNS friends from participant profiles. The moderator effects of these context variables were rarely examined in empirical studies and this meta-analysis tested the possibilities.

\section{Demographic Moderators}

Few studies have examined the effect of demographic variables, including participant gender and age, on the correlations of OSNS with well-being and distress. One exception was by Bandyopadhyay (2016), who sampled 100 male and 100 female undergraduate students and found that the coefficients for the correlation between selfesteem and the number of Facebook friends were .25 and .13 , respectively.

\section{Previous Meta-Analyses}

Pinquart and Sörensen (2000) synthesized the correlation between social network and subjective well-being for older adults with a mean age over 55 years. Those authors included studies investigating both quantitative and qualitative measures of social networks. The quantitative measures of social networks referred to the social network size and the frequency of social communication, while the qualitative measures referred to social support. The analysis identified 295 correlations between social network and life satisfaction, and obtained a weighted mean correlation coefficient of $r=.15$. The mean coefficient for the correlation between the social network and self-esteem was $r=.11(k=63)$, and that between social network and happiness was $r=.18(k=78)$. However, that meta-analysis was limited in three ways. First, only positive indicators were investigated, and distress indicators were not examined. Second, it included studies examining older adults, but none examining children, adolescents or young adults, so its research findings cannot necessarily be generalized to younger populations. Third, the study focused on offline social networks. Since both the quality and size of offline and online social networks can be different, those research findings might not be generalizable to online social networks.

D. Liu and Baumeister (2016) identified 11 studies on the correlation between the number of SNS friends and selfesteem, and found a mean correlation coefficient of .07. Since the number of effect sizes was small, the moderator effects on the correlation were not examined. 


\section{Purpose}

Online friends can be different from real-life friends (Perry et al., 2018). Therefore, the links of OSNS with wellbeing and distress might be different from those for offline social network size. Estimating the strength of correlations of OSNS with well-being and distress can help to determine whether online social network is beneficial or detrimental, and to understand the degree of the impact. Yet, few meta-analyses have been undertaken to estimate the relations, and therefore a meta-analysis is warranted. The purpose of the present study was to determine the magnitude and direction of the correlations of OSNS with well-being and distress.

\section{Method}

\section{Literature Search}

The PsycINFO, ERIC, and ProQuest Dissertations and Theses Global databases were searched using a combination of terms related to SNSs (namely, Facebook, Twitter, Instagram, MySpace, social media, online social network*, and social network* site*) and terms related to well-being and distress (namely, self-concept, self-esteem, selfworth, depress*, loneliness, life satisfaction, happ*, "mental health", anxi*, and distress*) through to May 24, 2019. The reference lists for all relevant articles as well as previous review articles (D. A. Baker \& Algorta, 2016; Best et al., 2014; Dobrean \& Păsărelu, 2016; Frost \& Rickwood, 2017; Huang, 2017; Keles et al., 2020; D. Liu \& Baumeister, 2016; Seabrook et al., 2016; Song et al., 2014; Vahedi \& Zannella, 2019; Yoon et al., 2019) were subsequently searched for additional relevant studies that were not identified in the computer-based searches. Articles were initially screened to obtain full texts by reading their titles and abstracts. The full texts were then reviewed to determine eligibility based on the following inclusion criteria: (1) reporting at least one of the correlations of the number of SNS friends with well-being and distress; (2) reporting the sample size so that weighted mean correlations could be calculated; (3) the level of well-being and distress being reported by the participant, with studies assessing well-being or distress using other sources excluded (e.g., the study by Moreno et al. (2011) that coded participant Facebook profiles to indicate depression); and (4) published in English.

\section{Dependence}

All correlations of OSNS with well-being and distress indicators were coded. Since this meta-analysis analyzed wellbeing and distress separately, each independent sample could contribute at most one correlation each for wellbeing and distress. To resolve the issue of dependence, the mean correlation was computed to correct for interdependent effect sizes for well-being and distress.

\section{Analysis}

Since the Pearson Product-Moment correlation coefficient $(r)$ has some undesirable properties (Lipsey \& Wilson, 2001), it was transformed into $Z_{r}$ using Fisher's transformation equation. The inverse variances were used as a weight to compute the weighted mean correlation coefficients. The weighted mean of $Z_{r}$ was then transformed back to correlation coefficient. The random-effects model is more general than the fixed-effect model. Moreover, type I error tends to be inflated in fixed-effect models (Hunter \& Schmidt, 2000). Therefore, this study used random-effects models. Comprehensive Meta-Analysis (Borenstein et al., 2013) was used to perform trim and fill examinations (Duval \& Tweedie, 2000) and other analyses were performed by MetaWin (M. S. Rosenberg et al., 2000).

To test whether the effect size distribution was homogenous, the $Q_{T}$ statistic was used, distributed approximately as $\chi^{2}$ with degrees of freedom equal to the number of effect sizes minus 1 . To test the effects of categorical moderators, the $Q_{B}$ statistic was used with degrees of freedom equal to the number of categories minus 1 . A significant $Q_{B}$ indicates heterogeneity among groups of effect sizes (Lipsey \& Wilson, 2001). 


\section{Results}

This meta-analysis included 90 articles involving 98 independent samples on correlations of the number of SNS friends with well-being and distress. These studies involved 33,329 participants, with a mean sample size of 340.09 participants and sample sizes range from 30 to 4,701 participants. The participants were assessed at ages of 13.30 and 20.92 years in Mikami et al. (2010); for the remaining cross-sectional studies, the mean age of the sample was available in 87 samples with a mean age of 24.47 years, ranging from 14 to 60 years. The mean social network size was available for 60 samples, with a weighted mean (by sample size) of 240.30 friends, ranging from 64 to 740 friends. Table 1 presents the summaries of the included studies.

Table 1. Summary of Studies on the Relation of Online Network Size With Well-Being and Distress.

\begin{tabular}{|c|c|c|c|c|c|c|c|c|c|c|}
\hline Study & $\mathrm{PO}$ & Country & $N$ & Age & Sex & WB/D & SNS & infoNS & Friends & $r$ \\
\hline Acar (2008) & J & US & 427 & 19.50 & .51 & $\begin{array}{l}\text { self-esteem, } \\
\text { anxiety }\end{array}$ & Facebook & $\begin{array}{c}\text { SR \& } \\
\text { PF }\end{array}$ & $217^{b}$ & $\begin{array}{l}.11 \\
-.08\end{array}$ \\
\hline $\begin{array}{l}\text { Ahmad et al. } \\
\text { (2016) }\end{array}$ & $\mathrm{J}$ & Pakistan & $461^{a}$ & 22.28 & .57 & self-esteem & SNS & SR & NA & $.05^{a}$ \\
\hline Arianna (2014) & $\mathrm{M}$ & miscellaneous & 147 & 25.16 & .70 & $\begin{array}{l}\text { self-esteem, } \\
\text { Ioneliness, } \\
\text { social } \\
\text { anxiety }\end{array}$ & Facebook & SR & 344.31 & $\begin{array}{l}.16 \\
-.27 \\
-.34\end{array}$ \\
\hline Ballinger (2018) & $\mathrm{D}$ & US & 97 & 59.90 & .77 & loneliness & Facebook & PF & NA & -.02 \\
\hline $\begin{array}{l}\text { Bandyopadhyay } \\
\text { (2016), \#1 }\end{array}$ & $\mathrm{M}$ & US & 100 & 19.59 & 0 & $\begin{array}{c}\text { self-esteem, } \\
\text { social } \\
\text { anxiety }\end{array}$ & Facebook & SR & 516.53 & $\begin{array}{l}.25 \\
-.17\end{array}$ \\
\hline $\begin{array}{l}\text { Bandyopadhyay } \\
\text { (2016), \#2 }\end{array}$ & $\mathrm{M}$ & US & 100 & 19.59 & 1 & $\begin{array}{l}\text { self-esteem, } \\
\text { social } \\
\text { anxiety }\end{array}$ & Facebook & SR & 738.77 & $\begin{array}{l}.13 \\
-.10\end{array}$ \\
\hline $\begin{array}{l}\text { Banjanin et al. } \\
\text { (2015) }\end{array}$ & $J$ & Serbia & 336 & 18 & .66 & depression & Facebook & SR & NA & .04 \\
\hline $\begin{array}{l}\text { Barry et al. } \\
\text { (2017) }\end{array}$ & $J$ & US & 128 & 20.46 & .85 & self-esteem & Instagram & PF & NA & $.13^{\mathrm{a}}$ \\
\hline $\begin{array}{l}\text { Bazarova et al. } \\
\text { (2017) }\end{array}$ & $J$ & US & 238 & 20.92 & .75 & $\begin{array}{l}\text { depression } \\
\& \text { anxiety }\end{array}$ & Facebook & NA & 739.92 & -.14 \\
\hline $\begin{array}{l}\text { Bevan-Dye } \\
\text { (2012) }\end{array}$ & $\mathrm{J}$ & South Africa & 346 & NA & .55 & self-esteem & Facebook & SR & NA & .12 \\
\hline Bourke (2013) & B & Ireland & $165 / 145 / 165$ & 13.66 & .57 & $\begin{array}{l}\text { self-esteem, } \\
\text { loneliness, } \\
\text { social } \\
\text { anxiety }\end{array}$ & Facebook & SR & 440.19 & $\begin{array}{l}.05 \\
.16 \\
-.18\end{array}$ \\
\hline Brailovskaia \& & & & & & & & & & & \\
\hline $\begin{array}{l}\text { Bierhoff (2020), } \\
\# 1\end{array}$ & J & Germany & 138 & 17.54 & .53 & self-esteem & Facebook & PF & 349.46 & .37 \\
\hline $\begin{array}{l}\text { Brailovskaia \& } \\
\text { Bierhoff (2020), } \\
\text { \#2 }\end{array}$ & J & Germany & 116 & 26.57 & .47 & self-esteem & Facebook & PF & 154.67 & .43 \\
\hline $\begin{array}{l}\text { Buglass et al. } \\
\text { (2017) }\end{array}$ & $J$ & UK & 506 & 20.58 & .47 & self-esteem & Facebook & SR & 424.28 & -.09 \\
\hline $\begin{array}{l}\text { Burrow \& } \\
\text { Rainone (2017) }\end{array}$ & $J$ & US & 246 & 32.63 & .49 & self-esteem & Facebook & SR & 371 & .13 \\
\hline $\begin{array}{l}\text { Carpenter } \\
(2012)\end{array}$ & $J$ & US & 294 & 23.26 & .68 & self-esteem & Facebook & SR & 652.58 & .04 \\
\hline $\begin{array}{l}\text { Chang et al. } \\
\text { (2015) }\end{array}$ & J & US & 577 & 42.64 & .59 & loneliness & Facebook & SR & 291.79 & .05 \\
\hline $\begin{array}{l}\text { Chen et al. } \\
\text { (2015) }\end{array}$ & J & miscellaneous & 352 & NA & .49 & self-esteem & Facebook & SR & NA & .05 \\
\hline $\begin{array}{l}\text { Chow \& Wan } \\
\text { (2017) }\end{array}$ & J & US & 282 & 33.19 & .30 & depression & Facebook & SR & 316.29 & 0 \\
\hline $\begin{array}{l}\text { Deatherage } \\
\text { (2016) }\end{array}$ & $\mathrm{D}$ & US & 208 & 20.50 & .63 & $\begin{array}{l}\text { loneliness, } \\
\text { life } \\
\text { satisfaction }\end{array}$ & Facebook & $S R$ & 563.4 & $\begin{array}{c}-.21 \\
.25\end{array}$ \\
\hline $\begin{array}{l}\text { Dumas et al. } \\
\text { (2017), \#1 }\end{array}$ & $J$ & US & 198 & 25 & .44 & Ioneliness & Instagram & SR & 307.53 & -.12 \\
\hline $\begin{array}{l}\text { Dumas et al. } \\
\text { (2017), \#2 }\end{array}$ & J & US & 265 & 25 & .47 & loneliness & Instagram & SR & 355.73 & .02 \\
\hline
\end{tabular}




\begin{tabular}{|c|c|c|c|c|c|c|c|c|c|c|}
\hline $\begin{array}{l}\text { Durak \& } \\
\text { Seferoğlu } \\
(2019)\end{array}$ & J & Turkey & 580 & NA & .60 & $\begin{array}{l}\text { loneliness, } \\
\text { social } \\
\text { anxiety }\end{array}$ & SNS & SR & 351 & $\begin{array}{l}-.10 \\
-.06\end{array}$ \\
\hline $\begin{array}{l}\text { Ellison et al. } \\
\text { (2007) }\end{array}$ & J & US & 286 & 20.10 & .66 & $\begin{array}{l}\text { self-esteem, } \\
\text { life } \\
\text { satisfaction }\end{array}$ & Facebook & SR & NA & $\begin{array}{l}.07^{\mathrm{a}} \\
.22^{\mathrm{a}}\end{array}$ \\
\hline $\begin{array}{l}\text { Fernandez et al. } \\
\text { (2012) }\end{array}$ & J & US & 62 & 19 & .63 & $\begin{array}{c}\text { depression, } \\
\text { social } \\
\text { anxiety }\end{array}$ & Facebook & PF & NA & $\begin{array}{l}.05 \\
-.45\end{array}$ \\
\hline $\begin{array}{l}\text { Flynn et al. } \\
\text { (2018) }\end{array}$ & J & miscellaneous & 715 & 31 & .81 & $\begin{array}{l}\text { depression, } \\
\text { anxiety, self- } \\
\text { esteem }\end{array}$ & Facebook & SR & 370 & $\begin{array}{l}.06 \\
.11 \\
-.03\end{array}$ \\
\hline $\begin{array}{l}\text { Gallagher } \\
\text { (2017) }\end{array}$ & M & US & 111 & 16.21 & .71 & self-esteem & SNS & $\mathrm{SR}$ & NA & .14 \\
\hline $\begin{array}{l}\text { Gerson et al. } \\
\text { (2016) }\end{array}$ & $\mathrm{J}$ & UK & 337 & 36.50 & .60 & $\begin{array}{c}\text { life } \\
\text { satisfaction }\end{array}$ & Facebook & $\mathrm{SR}$ & NA & $.13^{a}$ \\
\hline $\begin{array}{l}\text { Greitemeyer et } \\
\text { al. (2014), } \# 1\end{array}$ & $\mathrm{~J}$ & Austria & 458 & 22.70 & .68 & $\begin{array}{l}\text { Ioneliness, } \\
\text { self-esteem }\end{array}$ & Facebook & $\mathrm{SR}$ & 454 & $\begin{array}{l}-.13 \\
.03\end{array}$ \\
\hline $\begin{array}{l}\text { Greitemeyer et } \\
\text { al. (2014), \#2 }\end{array}$ & J & Austria & 1244 & NA & .73 & $\begin{array}{l}\text { loneliness, } \\
\text { self-esteem }\end{array}$ & Facebook & $\mathrm{SR}$ & 244 & $\begin{array}{c}-.11 \\
.04\end{array}$ \\
\hline Hill (2014) & $\mathrm{D}$ & US & 56 & NA & .41 & self-esteem & Facebook & SR & NA & .12 \\
\hline $\begin{array}{l}\text { Hollenbaugh \& } \\
\text { Ferris (2014) }\end{array}$ & $J$ & US & $285^{a}$ & 31.85 & .77 & self-esteem & Facebook & $\mathrm{SR}$ & 434.98 & $.01^{a}$ \\
\hline $\begin{array}{l}\text { Hong et al. } \\
\text { (2017) }\end{array}$ & J & US & $421^{a}$ & 34.79 & .62 & self-esteem & Facebook & $\mathrm{SR}$ & NA & $.04^{a}$ \\
\hline $\begin{array}{l}\text { Hood et al. } \\
\text { (2018) }\end{array}$ & $\mathrm{J}$ & Australia & 149 & 20.31 & .81 & $\begin{array}{c}\text { social } \\
\text { loneliness }\end{array}$ & SNS & $\mathrm{SR}$ & 172.64 & -.09 \\
\hline Jin (2013) & $\mathrm{J}$ & Korea & 536 & 34 & .50 & Ioneliness & Facebook & SR & 63.50 & -.13 \\
\hline $\begin{array}{l}\text { Kalpidou et al. } \\
\text { (2011) \#1 }\end{array}$ & $\mathrm{J}$ & US & 35 & 18.31 & .67 & self-esteem & Facebook & SR & NA & -.29 \\
\hline $\begin{array}{l}\text { Kalpidou et al. } \\
\text { (2011) \#2 }\end{array}$ & $\mathrm{J}$ & US & 35 & 20.91 & .67 & self-esteem & Facebook & SR & NA & -.04 \\
\hline $\begin{array}{l}\text { Kokkinos \& } \\
\text { Saripanidis } \\
(2017)\end{array}$ & J & Greece & $\begin{array}{c}227^{\mathrm{a}} / 232^{\mathrm{a}} \\
1232^{\mathrm{a}}\end{array}$ & 21.54 & .55 & $\begin{array}{l}\text { self-esteem, } \\
\text { depression, } \\
\text { loneliness }\end{array}$ & Facebook & $\mathrm{SR}$ & NA & $\begin{array}{l}-.07^{a} \\
.08^{a} \\
-.12^{a}\end{array}$ \\
\hline Krishnan (2011) & $\mathrm{D}$ & US & 674 & 19.64 & .56 & self-esteem & SNS & SR & 695.52 & .04 \\
\hline $\begin{array}{l}\text { Labrague } \\
\text { (2014) }\end{array}$ & $J$ & Philippines & 75 & 18.64 & .82 & $\begin{array}{l}\text { depression, } \\
\text { anxiety }\end{array}$ & Facebook & SR & NA & $\begin{array}{l}.14 \\
.16\end{array}$ \\
\hline $\begin{array}{l}\text { Landauer } \\
\text { (2014) }\end{array}$ & $\mathrm{D}$ & US & 312 & 19.68 & .82 & $\begin{array}{l}\text { depression, } \\
\text { self-esteem }\end{array}$ & Facebook & SR & 600.64 & $\begin{array}{l}-.03 \\
.06\end{array}$ \\
\hline $\begin{array}{l}\text { LaRose et al. } \\
\text { (2011) }\end{array}$ & $C$ & US & 364 & 17.76 & .70 & $\begin{array}{l}\text { self-esteem, } \\
\text { loneliness }\end{array}$ & Facebook & SR & 499.93 & $\begin{array}{l}.04 \\
-.24\end{array}$ \\
\hline Lee et al. (2012) & J & US & 234 & 19.68 & .69 & self-esteem & Facebook & SR & 566.32 & -.12 \\
\hline $\begin{array}{l}\text { Lee \& Jang } \\
\text { (2019) }\end{array}$ & J & Korea & 708 & 37.90 & .50 & $\begin{array}{c}\text { social } \\
\text { anxiety }\end{array}$ & Facebook & SR & NA & -.00 \\
\hline $\begin{array}{l}\text { Lima et al. } \\
\text { (2017), \#1 }\end{array}$ & $\mathrm{J}$ & Portugal & $223^{a}$ & 46.40 & .44 & $\begin{array}{l}\text { self-esteem, } \\
\text { life } \\
\text { satisfaction, } \\
\text { loneliness }\end{array}$ & Facebook & SR & NA & $\begin{array}{l}.03^{\mathrm{a}} \\
.02^{\mathrm{a}} \\
-.02^{\mathrm{a}}\end{array}$ \\
\hline $\begin{array}{l}\text { Lima et al. } \\
\text { (2017), \#2 }\end{array}$ & $\mathrm{J}$ & Portugal & $770^{a}$ & 44.10 & .50 & $\begin{array}{l}\text { self-esteem, } \\
\text { life } \\
\text { satisfaction, } \\
\text { loneliness }\end{array}$ & Facebook & SR & NA & $\begin{array}{l}.06^{\mathrm{a}} \\
.03^{\mathrm{a}} \\
-.03^{\mathrm{a}}\end{array}$ \\
\hline $\begin{array}{l}\text { J. Liu et al. } \\
\text { (2016) }\end{array}$ & J & US & 163 & 32.71 & .53 & self-esteem & Facebook & SR & NA & $-.14^{a}$ \\
\hline $\begin{array}{l}\text { Locatelli et al. } \\
(2012)\end{array}$ & J & US & 251 & 18.72 & .72 & $\begin{array}{c}\text { life } \\
\text { satisfaction, } \\
\text { depression }\end{array}$ & Facebook & SR & 585.30 & $\begin{array}{l}.01 \\
.06\end{array}$ \\
\hline Long (2012) & PP & US & 53 & 15.20 & .61 & depression & Facebook & SR & 325.70 & .12 \\
\hline $\begin{array}{l}\text { Lönnqvist \& } \\
\text { Deters (2016), } \\
\text { \#1 }\end{array}$ & $J$ & US & 153 & 20.20 & .61 & $\begin{array}{l}\text { happiness, } \\
\text { life } \\
\text { satisfaction }\end{array}$ & Facebook & PF & 523.30 & $\begin{array}{l}.21 \\
.29\end{array}$ \\
\hline $\begin{array}{l}\text { Lönnqvist \& } \\
\text { Deters (2016), } \\
\text { \#2 }\end{array}$ & $J$ & Germany & 187 & 23.50 & .79 & $\begin{array}{l}\text { happiness, } \\
\text { life } \\
\text { satisfaction }\end{array}$ & Facebook & PF & 213.60 & $\begin{array}{l}.16 \\
.15\end{array}$ \\
\hline
\end{tabular}




\begin{tabular}{|c|c|c|c|c|c|c|c|c|c|c|}
\hline & & & & & & & & & & \\
\hline $\begin{array}{l}\text { Lönnqvist \& } \\
\text { Itkonen (2014) }\end{array}$ & $J$ & Finland & 4701 & 32.60 & .66 & $\begin{array}{l}\text { satisfaction, } \\
\text { happiness }\end{array}$ & Facebook & PF & 251.93 & $\begin{array}{l}.08 \\
.09\end{array}$ \\
\hline $\begin{array}{l}\text { Manago et al. } \\
\text { (2012) }\end{array}$ & $J$ & US & 69 & 20.64 & .76 & $\begin{array}{c}\text { life } \\
\text { satisfaction, } \\
\text { self-esteem }\end{array}$ & Facebook & $\mathrm{SR}$ & 440 & $\begin{array}{l}.29 \\
.13\end{array}$ \\
\hline $\begin{array}{l}\text { Marshall et al. } \\
\text { (2015) }\end{array}$ & $J$ & US & $514^{a}$ & 30.90 & .59 & self-esteem & Facebook & SR & NA & $.03^{\mathrm{a}}$ \\
\hline Mazurek (2013) & J & US & 108 & 32.40 & .47 & loneliness & SNS & SR & 219.62 & $-.12^{\mathrm{a}}$ \\
\hline $\begin{array}{l}\text { Mersin \& Acılar } \\
(2015)\end{array}$ & J & Turkey & 696 & 21.99 & .70 & self-esteem & Facebook & $\mathrm{SR}$ & NA & -.00 \\
\hline $\begin{array}{l}\text { Metzler \& } \\
\text { Scheithauer } \\
\text { (2017) }\end{array}$ & J & Germany & 217 & 16.70 & .68 & self-esteem & Facebook & SR & 290 & .22 \\
\hline $\begin{array}{l}\text { Mikami et al. } \\
\text { (2010) }\end{array}$ & J & US & 92 & $\begin{array}{c}13.30 \\
\& \\
20.92\end{array}$ & .58 & depression & $\begin{array}{c}\text { Facebook \& } \\
\text { MySpace }\end{array}$ & PF & 298.60 & -.11 \\
\hline $\begin{array}{l}\text { Moorman } \\
\text { (2012) }\end{array}$ & $\mathrm{M}$ & Canada & 431 & 20.40 & .71 & depression & Facebook & $\mathrm{SR}$ & NA & .15 \\
\hline Morgan (2010) & D & US & 79 & 32.30 & .23 & $\begin{array}{l}\text { depression, } \\
\text { life } \\
\text { satisfaction }\end{array}$ & Facebook & SR & NA & $\begin{array}{l}-.12 \\
-.01\end{array}$ \\
\hline $\begin{array}{l}\text { Morin-Major et } \\
\text { al. (2016) }\end{array}$ & $J$ & Canada & 94 & 14.50 & .53 & $\begin{array}{l}\text { depression, } \\
\text { self-esteem }\end{array}$ & Facebook & $\mathrm{SR}$ & 124 & $\begin{array}{c}-.03 \\
.18\end{array}$ \\
\hline Murphy (2013) & $\mathrm{HD}$ & Ireland & 167 & 15.07 & .57 & $\begin{array}{c}\text { distress, } \\
\text { self-esteem }\end{array}$ & Facebook & SR & 463.47 & $\begin{array}{l}.04 \\
-.10\end{array}$ \\
\hline $\begin{array}{l}\text { Nabi et al. } \\
\text { (2013) }\end{array}$ & $J$ & US & 401 & 19.90 & .78 & $\begin{array}{c}\text { life } \\
\text { satisfaction }\end{array}$ & Facebook & SR & 375 & .20 \\
\hline Oh et al. (2014) & $J$ & US & 295 & 28 & .51 & $\begin{array}{c}\text { life } \\
\text { satisfaction }\end{array}$ & SNS & SR & 574.26 & .09 \\
\hline $\begin{array}{l}\text { Ophir et al. } \\
\text { (2019) }\end{array}$ & $J$ & Israel & 162 & NA & .51 & depression & Facebook & PF & 534.26 & .14 \\
\hline $\begin{array}{l}\text { S. Park et al. } \\
\text { (2015) }\end{array}$ & $C$ & Korea & 212 & 25.61 & .24 & depression & Facebook & PF & NA & -.25 \\
\hline $\begin{array}{l}\text { S. Y. Park \& } \\
\text { Baek (2018) }\end{array}$ & $J$ & Korea & 331 & 32.05 & .53 & $\begin{array}{c}\text { life } \\
\text { satisfaction }\end{array}$ & Facebook & $\mathrm{SR}$ & 148.43 & $.10^{\mathrm{a}}$ \\
\hline $\begin{array}{l}\text { Petrocchi et al. } \\
\text { (2015) }\end{array}$ & J & US & 205 & 20.50 & NA & Ioneliness & Facebook & $\mathrm{SR}$ & 583.70 & -.04 \\
\hline $\begin{array}{l}\text { Phu \& Gow } \\
\text { (2019) }\end{array}$ & J & UK & 332 & 21.54 & .71 & $\begin{array}{l}\text { happiness, } \\
\text { loneliness }\end{array}$ & Facebook & $\mathrm{SR}$ & 623 & $\begin{array}{l}.22 \\
-.21\end{array}$ \\
\hline $\begin{array}{l}\text { Reinecke \& } \\
\text { Trepte (2014) }\end{array}$ & J & Germany & 374 & 26 & .62 & $\begin{array}{c}\text { life } \\
\text { satisfaction }\end{array}$ & SNS & NA & 138.83 & $.12^{\mathrm{a}}$ \\
\hline Rogers (2017) & M & US & 30 & 15.02 & .30 & $\begin{array}{l}\text { depression, } \\
\text { self-esteem }\end{array}$ & Facebook & $\mathrm{SR}$ & 615.90 & $\begin{array}{l}.01 \\
.10\end{array}$ \\
\hline $\begin{array}{l}\text { Rosen et al. } \\
\text { (2013) }\end{array}$ & $J$ & US & $943^{a}$ & 30.74 & .60 & depression & Facebook & $\mathrm{SR}$ & NA & $-.05^{a}$ \\
\hline Schwartz (2010) & $D$ & US & 213 & 21 & .60 & $\begin{array}{l}\text { self-esteem, } \\
\text { Ioneliness }\end{array}$ & Facebook & $\mathrm{SR}$ & NA & $\begin{array}{l}.00 \\
-.01\end{array}$ \\
\hline Seo et al. (2016) & J & Korea & 285 & 21.81 & .39 & loneliness & Facebook & PF & 303.08 & $-.17^{a}$ \\
\hline Seto (2012) & $\mathrm{HP}$ & US & 175 & 19.20 & .84 & self-esteem & Facebook & SR & 702.56 & -.04 \\
\hline $\begin{array}{l}\text { Shchebetenko } \\
\text { (2019) }\end{array}$ & J & Russia & $829^{a}$ & 19.59 & .72 & self-esteem & VK.com & $\mathrm{SR}$ & 244.69 & $.11^{a}$ \\
\hline Sheldon (2012) & $J$ & US & 327 & 27 & .62 & Ioneliness & Facebook & SR & 447 & $-.18^{a}$ \\
\hline $\begin{array}{l}\text { Sherlock \& } \\
\text { Wagstaff (2019) }\end{array}$ & $J$ & Australia & 129 & 24.60 & 1 & $\begin{array}{l}\text { depression, } \\
\text { anxiety }\end{array}$ & Instagram & $\mathrm{SR}$ & NA & $\begin{array}{l}.22 \\
.28\end{array}$ \\
\hline Singh (2014) & M & US & 56 & NA & .59 & $\begin{array}{l}\text { self-esteem, } \\
\text { loneliness }\end{array}$ & Facebook & $\mathrm{SR}$ & 499.82 & $\begin{array}{l}-.13 \\
.10\end{array}$ \\
\hline $\begin{array}{l}\text { Skues et al. } \\
(2012)\end{array}$ & $J$ & Australia & 393 & 20.59 & .76 & $\begin{array}{l}\text { self-esteem, } \\
\text { loneliness }\end{array}$ & Facebook & $\mathrm{SR}$ & 349.97 & $\begin{array}{l}.04 \\
-.17\end{array}$ \\
\hline $\begin{array}{l}\text { Tang \& } \\
\text { Livingston } \\
\text { (2012) }\end{array}$ & $C$ & Hong Kong & 257 & NA & .58 & $\begin{array}{l}\text { loneliness, } \\
\text { depression }\end{array}$ & Facebook & $\mathrm{SR}$ & 552 & $\begin{array}{l}-.19 \\
-.07\end{array}$ \\
\hline $\begin{array}{l}\text { Turel \& Bechara } \\
\text { (2016) }\end{array}$ & J & US & 457 & 23.40 & .51 & self-esteem & Facebook & $\mathrm{SR}$ & 339.87 & .08 \\
\hline $\begin{array}{l}\text { Turel \& Qahri- } \\
\text { Saremi (2016) }\end{array}$ & $J$ & US & 341 & 23 & .52 & self-esteem & Facebook & SR & 348 & .11 \\
\hline $\begin{array}{l}\text { Utz et al. (2012), } \\
\# 1\end{array}$ & $J$ & Netherlands & 255 & 23.70 & .65 & self-esteem & SNS & $\mathrm{SR}$ & 224.89 & 0 \\
\hline
\end{tabular}




\begin{tabular}{|c|c|c|c|c|c|c|c|c|c|c|}
\hline $\begin{array}{l}\text { Utz et al. (2012), } \\
\# 2\end{array}$ & $J$ & Netherlands & 198 & 21.40 & .73 & self-esteem & SNS & $\mathrm{SR}$ & 220.80 & .04 \\
\hline $\begin{array}{l}\text { Vanman et al. } \\
\text { (2018) }\end{array}$ & J & Australia & 138 & 22.43 & .63 & $\begin{array}{c}\text { life } \\
\text { satisfaction }\end{array}$ & Facebook & $\mathrm{SR}$ & 506.11 & .11 \\
\hline $\begin{array}{l}\text { Wang et al. } \\
(2018)\end{array}$ & J & China & 325 & 32.67 & .54 & self-esteem & WeChat Moments & SR & NA & .22 \\
\hline $\begin{array}{l}\text { Weidman \& } \\
\text { Levinson (2015) }\end{array}$ & J & US & 77 & 18.91 & .77 & $\begin{array}{c}\text { social } \\
\text { anxiety }\end{array}$ & Facebook & $\mathrm{PF}$ & NA & -.36 \\
\hline $\begin{array}{l}\text { Wilcox \& } \\
\text { Stephen (2013) }\end{array}$ & J & US & $380^{a}$ & 29.85 & .56 & self-esteem & Facebook & $\mathrm{SR}$ & NA & $.13^{a}$ \\
\hline $\begin{array}{l}\text { Wright et al. } \\
\text { (2013) }\end{array}$ & J & US & 361 & 20.26 & .53 & depression & Facebook & $\mathrm{SR}$ & 560.09 & -.02 \\
\hline Yang (2016) & J & US & 208 & 19.43 & .78 & Ioneliness & Instagram & SR & NA & $-.10^{a}$ \\
\hline $\begin{array}{l}\text { Yang \& Brown } \\
\text { (2016) }\end{array}$ & J & US & 218 & 18.07 & .64 & self-esteem & Facebook & $\mathrm{SR}$ & NA & $.22^{\mathrm{a}}$ \\
\hline $\begin{array}{l}\text { Yoo \& Jeong } \\
\text { (2017) }\end{array}$ & J & Korea & 477 & NA & .43 & $\begin{array}{c}\text { life } \\
\text { satisfaction, } \\
\text { loneliness, } \\
\text { depression }\end{array}$ & SNS & SR & NA & $\begin{array}{l}.01 \\
-.18 \\
-.08\end{array}$ \\
\hline Young (2015) & M & US & 94 & 19.50 & .47 & self-esteem & Facebook & SR & NA & .22 \\
\hline $\begin{array}{l}\text { Zabawska } \\
\text { (2013) }\end{array}$ & B & Ireland & 123 & 33.74 & .57 & self-esteem & Facebook & $\mathrm{SR}$ & NA & .06 \\
\hline $\begin{array}{l}\text { Zeeni et al. } \\
\text { (2018) }\end{array}$ & J & Lebanon & 244 & 18.10 & .64 & $\begin{array}{c}\text { anxiety, } \\
\text { depression }\end{array}$ & Facebook/Instagram & NA & NA & $\begin{array}{l}-.01 \\
-.06\end{array}$ \\
\hline $\begin{array}{l}\text { Zell \& Moeller } \\
\text { (2018) }\end{array}$ & J & US & $307^{a}$ & 26.46 & .77 & self-esteem & Facebook & $\mathrm{SR}$ & 611.24 & $.03^{a}$ \\
\hline Zhang (2017) & $J$ & Hong Kong & 573 & NA & .60 & $\begin{array}{c}\text { depression, } \\
\text { life } \\
\text { satisfaction }\end{array}$ & Facebook & SR & NA & $\begin{array}{c}0 \\
-.03\end{array}$ \\
\hline
\end{tabular}

Notes. ${ }^{a}$ The information was not reported, but was obtained by contacting the authors.

${ }^{\mathrm{b}}$ The mean network size was computed based on 100 participants.

$N A=$ not available.

$\mathrm{PO}=$ publication outlet $\mathrm{J}=$ journal article, $\mathrm{B}=$ Bachelor's thesis, $\mathrm{M}=$ Master's thesis, $\mathrm{D}=$ Doctoral dissertation, $\mathrm{C}=$ conference paper, $\mathrm{HD}=\mathrm{Higher}$ Diploma, HP = thesis for honor program, PP = Master's professional paper; age = Mean age of the sample; Sex = proportion of females; $W B / D=$ indicator of well-being and distress; InfoNS = information about social network size, SR = self-report, PF = the number of SNS friends were obtained from the participant profile; friend = the mean number of SNS friends.

\section{Outlier Analyses}

Table 2 presents unweighted mean, standard deviation, minimum and maximum values of the Pearson ProductMoment correlation of each indicator with OSNS. The correlation between OSNS and self-esteem in the freshman sample ( $r=-.29$ ) of Kalpidou and colleagues (2011) was 2 standard deviations below the unweighted mean. As shown in Table 3, the weighted mean correlation was $r=.06$. When this study was excluded, the weighted mean correlation changed to $r=.07$. The effect size $(r=.43)$ reported in the early millennial sample of Brailovskaia and Bierhoff (2020) was 2 standard deviations above the unweighted mean. The weighted mean correlation between OSNS and self-esteem remained the same when that study was excluded. For depression, the effect size $(r=-.25)$ in S. Park et al. (2015) was 2 standard deviations below the unweighted mean. The weighted mean increased from $r=.01$ to .02 when that study was excluded. Bourke (2013) reported the correlation between OSNS and loneliness as $r=.16$, which is 2 standard deviations above the unweighted mean. The weighted mean remained the same $(r=-.11)$ when that study was excluded. As the influence of these extreme values was minimal, they remained in the subsequent analyses. Extreme values were not observed for other indicators.

Table 2. Summary Statistics for Effect Sizes.

\begin{tabular}{lcccc}
\hline & $r$ & $S D$ & Min & Max \\
\hline Happiness & .17 & .06 & .09 & .22 \\
Life satisfaction & .11 & .10 & -.03 & .29 \\
Self-esteem & .07 & .12 & -.29 & .43 \\
Anxiety & .09 & .14 & -.08 & .28 \\
Social anxiety & -.21 & .16 & -.45 & .00 \\
Depression & .01 & .11 & -.25 & .22 \\
Loneliness & -.10 & .11 & -.27 & .16 \\
\hline
\end{tabular}


Table 3. Summary of the Relations Between the Number of SNS Friends and Well-Being and Distress.

\begin{tabular}{lccccc}
\hline & $k$ & $r$ & \multicolumn{3}{c}{$95 \%$} \\
\cline { 5 - 6 } & & & lower & upper \\
\hline Well-being & 69 & .08 & .05 & .10 & 85.52 \\
Happiness & 4 & .15 & .02 & .28 & 1.95 \\
Life satisfaction & 18 & .10 & .06 & .15 & 21.02 \\
Self-esteem & 54 & .06 & .04 & .09 & 65.16 \\
Distress & 54 & -.06 & -.09 & -.03 & 57.08 \\
Anxiety & 5 & .08 & -.09 & .24 & 4.40 \\
Social anxiety & 8 & -.19 & -.32 & -.06 & 7.58 \\
Depression & 23 & .01 & -.04 & .05 & 23.25 \\
Loneliness & 26 & -.11 & -.14 & -.07 & 27.61 \\
\hline
\end{tabular}

Note. $Q_{T}=$ total homogeneity statistic.

\section{Publication Bias}

The effect of publication bias for each indicator with more than 1 effect size was tested by the trim and fill technique. No cases were imputed for self-esteem, anxiety, social anxiety, and loneliness. One effect size for life satisfaction was trimmed and yet the weighted mean effect size remained the same $(r=.10)$. Two effect sizes for happiness was trimmed and the mean correction decreased from $r=.15$ to .10 . Four effect sizes for depression were trimmed and the mean correction changed from $r=.01$ to -.02 .

\section{Mean Correlations}

Correlations between the number of SNS friends and well-being were reported in 69 samples and those between the number of SNS friends and distress in 54 samples. Coding multiple effect sizes for each sample yielded 141 correlations. The numbers of effect sizes were 1 for each of distress, combined anxiety and depression, and social loneliness, 5 effect sizes for anxiety, 23 for depression, 4 for happiness, 18 for life satisfaction, 26 for loneliness, 54 for self-esteem, and 8 for social anxiety. Table 3 lists the weighted mean correlations for indicators with more than one effect size. The mean correlations between the number of SNS friends and well-being indicators were positively weak based on the guidelines of Cohen (1988). Specifically, the correlations of SNS friends with wellbeing, happiness, life satisfaction, and self-esteem were $r=.08, .15, .10$, and .06 , respectively. The $95 \%$ confidence intervals did not include 0 , indicating that these correlations were significantly different from 0 . In contrast, the strengths of the correlations for distress indicators varied. For example, the correlation between OSNS and social anxiety was low-to-moderate and negative $(r=-.19)$, while the effect sizes for anxiety and depression did not differ significantly from 0 .

\section{Moderator Analyses}

Since general well-being, distress, and self-esteem had sufficiently large numbers of effect sizes, moderator analyses were conducted for these three indicators. When the moderators are categorical, the weighted mean of $Z_{r}$ for each category was computed and back transformed into the correlation coefficient.

\section{Well-Being}

Four categorical moderators were examined. As indicated in Table 4, most studies were reported in journals $(k=$ 51). The mean correlation between OSNS and well-being was significant for journal articles $(r=.08)$ and Master's theses $(r=.14)$. Publication outlet was not related to the correlation between the number of SNS friends and wellbeing. In contrast, the study country was significantly correlated with effect size. The correlation for studies conducted in Germany was moderate $(r=.24)$, while zero-to-low effect sizes were observed for the other countries. Most studies measured Facebook friends $(k=58)$, and found a low and positive relation between number of 
Facebook friends and well-being with $r=.07$. In contrast, the correlation between global online friends and wellbeing was not significantly different from $0(r=.06)$. The moderating effect of study SNS was not significant, with $Q_{B}=.31$. The number of SNS friends can be self-reported by the participant or obtained from the participant profile, and the method of obtaining information about the OSNS was significantly related to the correlation between OSNS and well-being, with $Q_{B}=12.27$. The mean correlation for studies using a self-report measure had a small effect size $(r=.06)$, while that for studies that recorded the OSNS from the participant profile was low-tomoderate, at $r=.21$.

Table 4. Moderator Analyses for Well-being

\begin{tabular}{|c|c|c|c|c|c|}
\hline \multirow{2}{*}{ Indicator } & \multirow{2}{*}{$k$} & \multirow{2}{*}{$r$} & \multicolumn{2}{|c|}{$95 \% \mathrm{Cl}$} & \multirow{2}{*}{$Q_{B}$} \\
\hline & & & upper & lower & \\
\hline \multicolumn{5}{|l|}{ Publication outlet } & 1.96 \\
\hline Journal & 51 & .08 & .05 & .10 & \\
\hline Bachelor & 2 & .05 & -.73 & .77 & \\
\hline Master & 7 & .14 & .03 & .26 & \\
\hline Doctor & 6 & .08 & -.03 & .18 & \\
\hline \multicolumn{5}{|l|}{ Country } & $16.60^{*}$ \\
\hline US & 36 & .08 & .04 & .11 & \\
\hline Ireland & 3 & -.00 & -.27 & .27 & \\
\hline Germany & 5 & .24 & .12 & .36 & \\
\hline UK & 3 & .08 & -.14 & .29 & \\
\hline Austria & 2 & .04 & -.60 & .65 & \\
\hline Portugal & 2 & .04 & -.64 & .68 & \\
\hline Korea & 2 & .05 & -.63 & .69 & \\
\hline Australia & 2 & .07 & -.67 & .74 & \\
\hline Netherlands & 2 & .02 & -.70 & .72 & \\
\hline \multicolumn{5}{|l|}{ Site } & 0.31 \\
\hline Facebook & 58 & .07 & .05 & .10 & \\
\hline SNS & 8 & .06 & -.02 & .13 & \\
\hline \multicolumn{5}{|c|}{ How the Information about the Social Network Size Was Obtained } & $12.27^{*}$ \\
\hline Self-report & 61 & .06 & .04 & .09 & \\
\hline Profile & 6 & .21 & .11 & .31 & \\
\hline
\end{tabular}

Notes. $Q_{B}=$ between-group homogeneity statistic; How the information about the social network size was obtained, profile $=$ the number of SNS friends were obtained from the participant profile. ${ }^{*} p<.05$.

Table 5. Effects of Continuous Moderators.

\begin{tabular}{lcccc}
\hline Variable & $k$ & $a^{a}$ & $b^{b}$ & $p$ \\
\hline Well-being & & & & \\
$\quad$ female & 69 & .12 & -.07 & .44 \\
$\quad$ mean age & 62 & .12 & -.00 & .35 \\
$\quad$ mean network size & 43 & .15 & -.00 & .10 \\
Distress & & & & \\
$\quad$ female & 53 & -.13 & .11 & .22 \\
$\quad$ mean age & $46^{a}$ & -.11 & .00 & .38 \\
$\quad$ mean network size & 35 & -.07 & .00 & .87 \\
Self-esteem & & & & \\
$\quad$ female & 54 & .17 & -.18 & .07 \\
$\quad$ mean age & 49 & .09 & -.00 & .58 \\
$\quad$ mean network size & 32 & .17 & -.00 & .02 \\
\hline
\end{tabular}




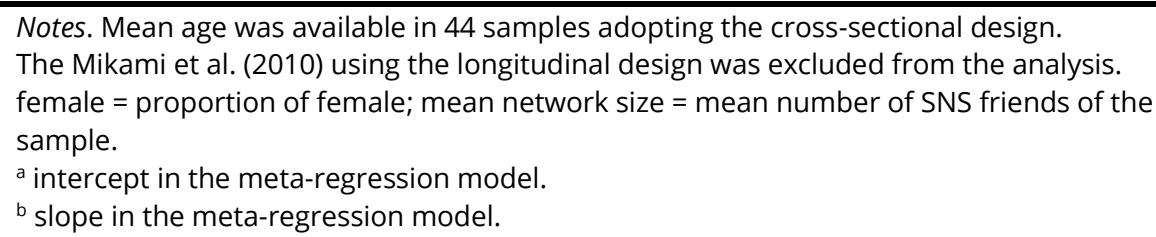

Table 5 presents the effects of continuous moderators, namely the proportion of females, mean age and mean OSNS of the sample. The proportion of females in the sample was available in 69 samples; mean age of the sample in 62 samples, and mean number of online friends in 43 samples. Since the $p$ values of regression coefficients $(b)$ were all larger than .05, the null hypotheses that regression coefficients were 0 were not rejected. Thus, all continuous moderators were unrelated to the correlation between OSNS and well-being.

\section{Distress}

Table 6 presents the categorical effects for the effect sizes of distress. The mean correlations for journal articles was significantly different from $0(r=-.05)$, while those for Master's theses, doctoral dissertations and conference papers were 0 . Again, most studies were conducted in US. All other means, except the weighted means for US $(r=-.08)$ and Korea $(r=-.13)$, were not significantly different from 0 . The non-significance may be caused by the low statistical power due to the small numbers of effect sizes. Facebook again attracted the most research attention, and the mean for Facebook friends was significantly different from 0. A self-report measure was the most popular method for measuring the OSNS. The weighted mean correlation between OSNS and distress was significantly different from 0 when measuring the OSNS by self-reporting $(r=-.05)$ or from participant profiles $(r=-.13)$. None of the categorical moderator analyses demonstrated significant effects on the correlation between OSNS and distress. As indicated in Table 5, $p$ values of regression coefficients were again larger than .05 , indicating that none of the continuous moderators were statistically significant.

Table 6. Moderator Analyses for Distress.

\begin{tabular}{|c|c|c|c|c|c|}
\hline \multirow{2}{*}{ Indicator } & \multirow{2}{*}{$k$} & \multirow{2}{*}{$r$} & \multicolumn{2}{|c|}{$95 \% \mathrm{Cl}$} & \multirow{2}{*}{$Q_{B}$} \\
\hline & & & upper & lower & \\
\hline \multicolumn{5}{|l|}{ Publication outlet } & 6.88 \\
\hline Journal & 37 & -.05 & -.08 & -.01 & \\
\hline Master & 6 & -.05 & -.19 & .08 & \\
\hline Doctor & 5 & -.08 & -.22 & .07 & \\
\hline Conference & 3 & -.21 & -.44 & .05 & \\
\hline \multicolumn{5}{|l|}{ Country } & 9.63 \\
\hline US & 27 & -.08 & -.12 & -.03 & \\
\hline Ireland & 2 & .02 & -.75 & .77 & \\
\hline Austria & 2 & -.12 & -.71 & .57 & \\
\hline Australia & 3 & -.03 & -.29 & .22 & \\
\hline Korea & 5 & -.13 & -.24 & -.01 & \\
\hline Portugal & 2 & -.02 & -.69 & .66 & \\
\hline Canada & 2 & .09 & -.69 & .77 & \\
\hline Hong Kong & 2 & -.06 & -.71 & .65 & \\
\hline \multicolumn{5}{|l|}{ Site } & 1.21 \\
\hline Facebook & 44 & -.06 & -.10 & -.03 & \\
\hline Instagram & 4 & .00 & -.18 & .19 & \\
\hline SNS & 4 & -.05 & -.23 & .13 & \\
\hline \multicolumn{5}{|c|}{ How the information about the social network size was obtained } & 2.78 \\
\hline Self-report & 44 & -.05 & -.08 & -.01 & \\
\hline Profile & 7 & -.13 & -.25 & -.01 & \\
\hline
\end{tabular}

Note. $Q_{B}=$ between-group homogeneity statistic; How the information about the social network size was obtained, profile $=$ the number of SNS friends were obtained from the participant profile. 
Since all effect sizes for self-esteem were also included in those for well-being, the results of the categorical moderator analyses for self-esteem were similar to those for well-being. As indicated in Table 7, the mean correlations for publication outlets with relatively large numbers of effect sizes (journal articles and Master's theses) were significantly different from $0(r=.06$ and .14 , respectively), while those with small numbers of effect sizes (Bachelor's Theses and doctoral dissertations) were not $(r=.05)$. The effect of publication status was not significant, with $Q_{B}=2.74$. Conversely, the effect of country where the study was conducted was significant, with $Q_{B}=28.83$. The effect size was moderate for Germany $(r=.32)$, but zero-to-low for all of the other countries. The study SNS was not related to the correlation between OSNS and self-esteem with $Q_{B}=.09$. The effect of how the information of social network size obtained was significant. The mean effect size for self-report measures $(r=.05)$ was small, while mean correlation for that obtained from the participant profile was moderate $(r=.31)$. The patterns of continuous moderators of effect sizes for self-esteem and for well-being were somewhat different. The effect of the mean number of SNS friends of the sample was significant. The weighted regression equation was $Z_{\mathrm{r}}=.17+(-.0003) \times$ (mean social network size). The expected coefficients for the correlation between OSNS and self-esteem were about $r=.14, r=.11$, and $r=.08$ for samples with means of 100, 200, and 300 SNS friends, respectively. In other words, the correlation between OSNS and self-esteem weakened as the mean OSNS increased. The gender composition and mean age did not exert significant effects on the correlation between the number of SNS friends and self-esteem.

Table 7. Moderator Analyses for Self-Esteem.

\begin{tabular}{|c|c|c|c|c|c|}
\hline \multirow{2}{*}{ Indicator } & \multirow{2}{*}{$k$} & \multirow{2}{*}{$r$} & \multicolumn{2}{|c|}{$95 \% \mathrm{Cl}$} & \multirow{2}{*}{$Q_{B}$} \\
\hline & & & upper & lower & \\
\hline Publication outlet & & & & & 2.74 \\
\hline Journal & 38 & .06 & .03 & .10 & \\
\hline Bachelor & 2 & .05 & -.73 & .78 & \\
\hline Master & 7 & .14 & .02 & .26 & \\
\hline Doctor & 4 & .05 & -.11 & .20 & \\
\hline Country & & & & & $28.83^{\star \star \star}$ \\
\hline US & 30 & .06 & .03 & .09 & \\
\hline Ireland & 3 & -.00 & -.23 & .22 & \\
\hline Germany & 3 & .32 & .11 & .51 & \\
\hline Austria & 2 & .04 & -.44 & .50 & \\
\hline Portugal & 2 & .05 & -.49 & .56 & \\
\hline Netherlands & 2 & .02 & -.60 & .63 & \\
\hline Site & & & & & 0.09 \\
\hline Facebook & 46 & .06 & -.03 & .09 & \\
\hline SNS & 5 & .05 & -.07 & .16 & \\
\hline \multicolumn{5}{|c|}{ How the information about the social network size was obtained } & $19.07^{\star \star \star}$ \\
\hline Self-report & 50 & .05 & .03 & .08 & \\
\hline Profile & 3 & .31 & .06 & .53 & \\
\hline
\end{tabular}

Notes. How the information about the social network size was obtained, profile = the number of SNS friends were obtained from the participant profile.

${ }^{* * *} p<.001$.

\section{Discussion}

There has been a considerable amount of research into the effect of social network on health and distress (Santini et al., 2015; Smith \& Christakis, 2008). The purpose of the present meta-analysis was to estimate the directions and magnitudes of the correlations of OSNS with well-being and distress. Since most Facebook users are aged 1834 years (Statista, 2019), the present study provided crucial information for understanding the links of social network size with well-being and distress among adolescents and young adults.

To ensure the validity of findings of this study, the trim and fill method was performed to correct the effect of publication bias. No publication bias was found in most indicators. For the indicators of life satisfaction, happiness, and loneliness, the decrement of effect size ranged from .00 to .05 after the trim and fill techniques. Hence, the impact of publication bias was not substantial. 


\section{Overall Relations of the Number of SNS Friends With Well-Being and Distress}

Substantially meaningful relations between OSNS and well-being indicators were observed, as all the correlations were significant and positive. These findings indicated that larger OSNS was related to higher level of well-being. The most frequently examined indicator of well-being was self-esteem, and the coefficient for its correlation with OSNS was $r=.06$, which is comparable to D. Liu and Baumeister (2016) reporting a coefficient of $r=.07$. However, the current study had a smaller effect size than that of Pinquart and Sörensen (2000). The inter-study difference may be explained by variations in the sample ages, and in the indexes used to measure social networks. Pinquart and Sörensen (2000) included studies that examined offline social networks for participants with a mean age over 55 years. Furthermore, the social networks were characterized by the network size, communication frequency, and social support. The correlations of online network size with life satisfaction and happiness found in the present study were also weaker than those found by Pinquart and Sörensen (2000).

This study found mixed relations between OSNS and distress indicators. Correlations of loneliness and social anxiety with OSNS were negative, whereas the correlation coefficients for anxiety and depression did not differ significantly from 0 . Furthermore, social anxiety had the strongest correlation with OSNS, with a low-to-moderate effect size $(r=-.19)$. The direction and magnitude of the correlation between OSNS and distress indicators varied, and thus future research studies should include multiple distress indicators to help understand their links with OSNS.

\section{Moderator Effects}

The moderator analyses indicated that moderate correlations between OSNS and well-being were found in studies conducted in Germany. Further examination revealed that two samples from Brailovskaia and Bierhoff (2020) had unusually strong correlations $(r=.37$ and $r=.43$ ). The mean correlation coefficient for Germany after these two potential outliers were removed was $r=.16$, which was still larger than those for the other countries. Since the moderator analyses for well-being also included all effect sizes for self-esteem, the effect of study country on the correlation between OSNS and self-esteem was also significant. Two of the three German samples assessing selfesteem were from Brailovskaia and Bierhoff (2020). Other countries, except US, had smaller numbers of samples, and more research into the country effect is needed.

The size of an online social network can be accurately determined from the participant profile. The use of this measure yielded an about moderate effect size for self-esteem, while that for the self-report measure was small. This finding indicated that magnitude of the link between OSNS and self-esteem depends on accuracy of the measure used to quantify the OSNS. The use of a proxy measure of OSNS underestimated the strength of the correlation. Hence, future research should adopt an accurate measure, rather than a proxy measure, when attempting to precisely determine the magnitude of correlations.

The mean social network size was related to the correlation between OSNS and self-esteem. Studies involving smaller social networks yielded stronger correlations than those involving larger social networks. Weak relationships, such as those that are common on online social networks (Donath \& boyd, 2004), may replace strong relationships in samples involving extremely large networks. This possibility suggests that more research is needed into the correlations of the number of close SNS friends with well-being and distress (e.g., Lemieux et al., 2013). Further research should also consider other potential moderators, such as representativeness of sample, measure of research variable (multi-item versus single-item scale) and socio-economic status.

Although the meta-analysis by Pinquart and Sörensen (2000) reported the mean correlation between social network and subjective well-being, they focused on the relations between offline social networks and positive indicators for older adults. D. Liu and Baumeister (2016) focused on online social networks, but only included a small number of studies, and only examined self-esteem as an indicator. This meta-analysis contributes beyond previous meta-analyses because of its research scope and addition of indicators of well-being and distress. This study shifts the research scope to offline social network and to participants from all age groups, and incorporates several positive and negative indicators to provide a comprehensive assessment of mental health. 


\section{Limitations}

This study has some limitations. First, this study focused on the size of social networks, and so does not determine the correlations between the quality of online social network with well-being and distress. Second, because the establishment of criteria to determine study quality of primary research is difficult in correlational research (Lipsey \& Wilson, 2001; Valentine 2009), the moderator of study quality was not examined. Lastly, the effects of well-being and distress measures were not examined, because the same scales have been widely used to measure selfesteem, loneliness and life satisfaction. Specifically, the Rosenberg Self-esteem Scale (M. Rosenberg, 1965) is frequently used to measure self-esteem, the UCLA Loneliness Scale (Russell, 1996) is used for measuring loneliness, and the Satisfaction with Life Scale (Diener et al., 1985) is used to assess life satisfaction. Very few studies have used different measures, so this study did not investigate the moderator effects of measures.

\section{References}

References marked with an asterisk indicate studies included in the meta-analysis.

*Acar, A. (2008). Antecedents and consequences of online social networking behavior: The case of Facebook. Journal of Website Promotion, 3(1-2), 62-83. https://doi.org/10.1080/15533610802052654

*Ahmad, S., Mustafa, M., \& Ullah, A. (2016). Association of demographics, motives and intensity of using social networking sites with the formation of bonding and bridging social capital in Pakistan. Computers in Human Behavior, 57, 107-114. https://doi.org/10.1016/j.chb.2015.12.027

Antonucci, T. C., Ajrouch, K. J., \& Birditt, K. S. (2014). The convoy model: Explaining social relations from a multidisciplinary perspective. The Gerontologist, 54(1), 82-92. https://doi.org/10.1093/geront/gnt118

*Arianna, P. (2014). Online self-presentation: Examining the relationship between Facebook use, narcissism, social anxiety, loneliness and self-esteem [Unpublished master's thesis]. Glasgow Caledonian University.

Augusteijn, H. E. M., van Aert, R. C. M., \& van Assen, M. A. L. M. (2019). The effect of publication bias on the Q test and assessment of heterogeneity. Psychological Methods, 24(1), 116-134. https://doi.org/10.1037/met0000197

Baker, D. A., \& Algorta, G. P. (2016). The relationship between online social networking and depression: A systematic review of quantitative studies. Cyberpsychology, Behavior, and Social Networking, 19(11), 638-648. https://doi.org/10.1089/cyber.2016.0206

Baker, L. R., \& Oswald, D. L. (2010). Shyness and online social networking services. Journal of Social and Personal Relationships, 27(7), 873-889. https://doi.org/10.1177/0265407510375261

*Ballinger, L. E. (2018). Understanding socialization efficacy and loneliness of baby boomers through Facebook [Doctoral dissertation, Walden University]. Walden Dissertations and Doctoral Studies.

https://scholarworks.waldenu.edu/dissertations/4906

*Bandyopadhyay, P. (2016). The relationship between Facebook friends and self-esteem [Unpublished master's thesis]. University of Texas at San Antonio.

*Banjanin, N., Banjanin, N., Dimitrijevic, I., \& Pantic, I. (2015). Relationship between internet use and depression: Focus on physiological mood oscillations, social networking and online addictive behavior. Computers in Human Behavior, 43, 308-312. https://doi.org/10.1016/j.chb.2014.11.013

*Barry, C. T., Doucette, H., Loflin, D. C., Rivera-Hudson, N., \& Herrington, L. L. (2017). "Let me take a selfie": Associations between self-photography, narcissism, and self-esteem. Psychology of Popular Media Culture, 6(1), 48-60. https://doi.org/10.1037/ppm0000089 
*Bazarova, N. N., Choi, Y. H., Whitlock, J., Cosley, D., \& Sosik, V. (2017). Psychological distress and emotional expression on Facebook. Cyberpsychology, Behavior, and Social Networking, 20(3), 157-163.

https://doi.org/10.1089/cyber.2016.0335

Best, P., Manktelow, R., \& Taylor, B. (2014). Online communication, social media and adolescent wellbeing: A systematic narrative review. Children and Youth Services Review, 41, 27-36.

https://doi.org/10.1016/j.childyouth.2014.03.001

*Bevan-Dye, A. L. (2012). Relationship between self-esteem and Facebook usage amongst black Generation Y students. African Journal for Physical Health Education, Recreation and Dance, 18(Suppl. 1), 33-49.

https://hdl.handle.net/10520/EJC139747

Borenstein, M., Hedges, L., Higgins, J., \& Rothstein, H (2013). Comprehensive meta-analysis. Biostat.

*Bourke, N. (2013). Online social networking and well-being in adolescents [Unpublished bachelor's thesis]. Dublin Business School.

*Brailovskaia, J., \& Bierhoff, H.-W. (2020). The narcissistic millennial generation: A study of personality traits and online behavior on Facebook. Journal of Adult Development, 27(1), 23-35. https://doi.org/10.1007/s10804-018-

$9321-1$

*Buglass, S. L., Binder, J. F., Betts, L. R., \& Underwood, J. D. M. (2017). Motivators of online vulnerability: The impact of social network site use and FOMO. Computers in Human Behavior, 66, 248-255.

https://doi.org/10.1016/j.chb.2016.09.055

*Burrow, A. L., \& Rainone, N. (2017). How many likes did I get? Purpose moderates links between positive social media feedback and self-esteem. Journal of Experimental Social Psychology, 69, 232-236.

https://doi.org/10.1016/j.jesp.2016.09.005

*Carpenter, C. J. (2012). Narcissism on Facebook: Self-promotional and anti-social behavior. Personality and Individual Differences, 52(4), 482-486. https://doi.org/10.1016/j.paid.2011.11.011

Carstensen, L. L. (1995). Evidence for a life-span theory of socioemotional selectivity. Current Directions in Psychological Science, 4(5), 151-156. https://doi.org/10.1111/1467-8721.ep11512261

Carstensen, L. L., Isaacowitz, D. M., \& Charles, S. T. (1999). Taking time seriously: A theory of socioemotional selectivity. American Psychologist, 54(3), 165-181. https://doi.org/10.1037/0003-066X.54.3.165

Chan, Y. K., \& Lee, R. P. L. (2006). Network size, social support and happiness in later life: A comparative study of Beijing and Hong Kong. Journal of Happiness Studies, 7(1), 87-112. https://doi.org/10.1007/s10902-005-1915-1

*Chang, P. F., Choi, Y. H., Bazarova, N. N., \& Löckenhoff, C. E. (2015). Age differences in online social networking: Extending socioemotional selectivity theory to social network sites. Journal of Broadcasting \& Electronic Media, 59(2), 221-239. https://doi.org/10.1080/08838151.2015.1029126

*Chen, J. V., Widjaja, A. E., \& Yen, D. C. (2015). Need for affiliation, need for popularity, self-esteem, and the moderating effect of Big Five personality traits affecting individuals' self-disclosure on Facebook. International Journal of Human-Computer Interaction, 31(11), 815-831. https://doi.org/10.1080/10447318.2015.1067479

${ }^{*}$ Chow, T. S., \& Wan, H. Y. (2017). Is there any 'Facebook Depression'? Exploring the moderating roles of neuroticism, Facebook social comparison and envy. Personality and Individual Differences, 119, 277-282. https://doi.org/10.1016/j.paid.2017.07.032

Cohen, J. (1988). Statistical power analysis for the behavioral sciences (2nd ed.). Routledge. 
*Deatherage, S. S. (2016). Facebook engagement on college students' interpersonal and intrapersonal functioning [Doctoral dissertation, Purdue University]. https://docs.lib.purdue.edu/open_access_dissertations/748

Deci, E. L, \& Ryan, R. M. (2008). Hedonia, eudaimonia, and well-being: An introduction. Journal of Happiness Studies, 9(1), 1-11. https://doi.org/10.1007/s10902-006-9018-1

Diener E. (2000). Subjective well-being: The science of happiness and a proposal for a national index. American Psychologist, 55(1), 34-43. https://doi.org/10.1037/0003-066X.55.1.34

Diener, E., Emmons, R. A., Larsen, R. J., \& Griffin, S. (1985). The Satisfaction With Life Scale. Journal of Personality Assessment, 49(1), 71-75. https://doi.org/10.1207/s15327752jpa4901_13

Dobrean, A., \& Păsărelu, C.-R. (2016). Impact of social media on social anxiety: A systematic review. In F. Durbano (Ed.), New developments in anxiety disorders. IntechOpen.

Domènech-Abella, J., Lara, E., Rubio-Valera, M., Olaya, B., Moneta, M. V., Rico-Uribe, L. A., Ayuso-Mateos, J. L., Mundó, J., \& Haro, J. M. (2017). Loneliness and depression in the elderly: The role of social network. Social Psychiatry and Psychiatric Epidemiology, 52(4), 381-390. https://doi.org/10.1007/s00127-017-1339-3

Donath, J., \& boyd, d. (2004). Public displays of connection. BT Technology Journal, 22(4), 71-82.

https://doi.org/10.1023/B:BTTJ.0000047585.06264.cc

*Dumas, T. M., Maxwell-Smith, M., Davis, J. P., \& Giulietti, P. A. (2017). Lying or longing for likes? Narcissism, peer belonging, loneliness and normative versus deceptive like-seeking on Instagram in emerging adulthood.

Computers in Human Behavior, 71, 1-10. https://doi.org/10.1016/j.chb.2017.01.037

*Durak, H. Y., \& Seferoğlu, S. S. (2019). Modeling of variables related to problematic social media usage: Social desirability tendency example. Scandinavian Journal of Psychology, 60(3), 277-288.

https://doi.org/10.1111/sjop.12530

Duval, S., \& Tweedie, R. L. (2000). A non-parametric "trim and fill" method of accounting for publication bias in meta-analysis. Journal of the American Statistical Association, 95(449), 89-98.

https://doi.org/10.1080/01621459.2000.10473905

*Ellison, N. B., Steinfield, C., \& Lampe, C. (2007). The benefits of Facebook "friends:" Social capital and college students' use of online social network sites. Journal of Computer-Mediated Communication, 12(4), 1143-1168. https://doi.org/10.1111/j.1083-6101.2007.00367.x

Ferguson, C. J., \& Heene, M. (2012). A vast graveyard of undead theories: Publication bias and psychological science's aversion to the null. Perspectives on Psychological Science, 7(6), 555-561.

https://doi.org/10.1177/1745691612459059

*Fernandez, K. C., Levinson, C. A., \& Rodebaugh, T. L. (2012). Profiling: Predicting social anxiety from Facebook profiles. Social Psychological and Personality Science, 3(6), 706-713. https://doi.org/10.1177/1948550611434967

Fiori, K. L., Smith, J., \& Antonucci, T. C. (2007). Social network types among older adults: A multidimensional approach. Journals of Gerontology, 62(6), 322-330. https://doi,org/10.1093/geronb/62.6.P322

*Flynn, S., Noone, C., \& Sarma, K. M. (2018). An exploration of the link between adult attachment and problematic Facebook use. BMC Psychology, 6, Article 34. https://doi.org/10.1186/s40359-018-0245-0

Frost, R. L., \& Rickwood, D. J. (2017). A systematic review of the mental health outcomes associated with Facebook use. Computers in Human Behavior, 76, 576-600. https://doi.org/10.1016/j.chb.2017.08.001 
*Gallagher, S. M. (2017). The influence of social media on teens' self-esteem [Master's thesis, Rowan University]. https://rdw.rowan.edu/etd/2438

*Gerson, J., Plagnol, A. C., \& Corr, P. J. (2016). Subjective well-being and social media use: Do personality traits moderate the impact of social comparison on Facebook? Computers in Human Behavior, 63, 813-822.

https://doi.org/10.1016/j.chb.2016.06.023

*Greitemeyer, T., Mügge, D. O., \& Bollermann, I. (2014). Having responsive Facebook friends affects the satisfaction of psychological needs more than having many Facebook friends. Basic and Applied Social Psychology, 36(3), 252-258. https://doi.org/10.1080/01973533.2014.900619

*Hill, C. L. (2014). An investigation of the connections between use of Facebook and the self-esteem/well-being of students with disabilities in the University of lowa REACH program [Doctoral dissertation, University of lowa]. https://doi.org/10.17077/etd.5fxjx7dz

*Hollenbaugh, E. E., \& Ferris, A. L. (2014). Facebook self-disclosure: Examining the role of traits, social cohesion, and motives. Computers in Human Behavior, 30, 50-58. https://doi.org/10.1016/j.chb.2013.07.055

*Hong, C., Chen, Z. (F.), \& Li, C. (2017). "Liking" and being "liked": How are personality traits and demographics associated with giving and receiving "likes" on Facebook? Computers in Human Behavior, 68, 292-299. https://doi.org/10.1016/j.chb.2016.11.048

*Hood, M., Creed, P. A., \& Mills, B. J. (2018). Loneliness and online friendships in emerging adults. Personality and Individual Differences, 133, 96-102. https://doi.org/10.1016/j.paid.2017.03.045

Huang, C. (2017). Time spent on social network sites and psychological well-being: A meta-analysis. Cyberpsychology, Behavior, and Social Networking, 20(6), 346-354. https://doi.org/10.1089/cyber.2016.0758

Hunter, J. E., \& Schmidt, F. L. (2000). Fixed effects vs. random effects meta-analysis models: Implications for cumulative research knowledge. International Journal of Selection and Assessment, 8(4), 275-292.

https://doi.org/10.1111/1468-2389.00156

*Jin, B. (2013). How lonely people use and perceive Facebook. Computers in Human Behavior, 29(6), 2463-2470. https://doi.org/10.1016/j.chb.2013.05.034

*Kalpidou, M., Costin, D., \& Morris, J. (2011). The relationship between Facebook and the well-being of undergraduate college students. Cyberpsychology, Behavior, and Social Networking, 14(4), 183-189. https://doi.org/10.1089/cyber.2010.0061

Keles, B., McCrae, N., \& Grealish, A. (2020). A systematic review: The influence of social media on depression, anxiety and psychological distress in adolescents, International Journal of Adolescence and Youth, 25(1). 79-93. https://doi.org/10.1080/02673843.2019.1590851

Keyes, C. L. M. (2007). Promoting and protecting mental health as flourishing: A complementary strategy for improving national mental health. American Psychologist, 62(2), 95-108. https://doi.org/10.1037/0003-

066X.62.2.95

*Kokkinos, C. M., \& Saripanidis, I. (2017). A lifestyle exposure perspective of victimization through Facebook among university students. Do individual differences matter? Computers in Human Behavior, 74, 235-245. https://doi.org/10.1016/j.chb.2017.04.036

*Krishnan, A. (2011). Individual differences in users of online networking sites: The interplay between personality traits, communication and social motives, attitudes and level of activity [Doctoral dissertation, University of Connecticut]. ProQuest Dissertations and Theses Database. https://search.proquest.com/dissertations-theses/individualdifferences-users-online-networking/docview/882906957/se-2?accountid=16531 
*Labrague, J. L. (2014). Facebook use and adolescents' emotional states of depression, anxiety, and stress. Health Science Journal, 8(1), 80-89.

*Landauer, D. B. (2014). Depression, negative self-disclosure, and response of others on Facebook [Doctoral dissertation, University of North Dakota]. https://commons.und.edu/theses/1675

*LaRose, R., Wohn, D. Y., Ellison, N., \& Steinfield, C. (2011). Facebook fiends: Compulsive social networking and adjustment to college. In G. Bradley, D. Whitehouse, \& G. Singh (Eds.), ICT 2011: Proceedings of the International Association for the Development of the Information Society. IADIS.

*Lee, J. R., Moore, D. C., Park, E., \& Park, S. G. (2012). Who want to be "friend-rich"? Social compensatory friending on Facebook and the moderating role of public self-consciousness. Computers in Human Behavior, 28(3), 10361043. https://doi.org/10.1016/j.chb.2012.01.006

*Lee, S. Y., \& Jang, K. (2019). Antecedents of impression management motivations on social network sites and their link to social anxiety. Media Psychology, 22(6), 890-904. https://doi.org/10.1080/15213269.2019.1580588

Lemieux, R., Lajoie, S., \& Trainor, N. E. (2013). Affinity-seeking, social loneliness, and social avoidance among Facebook users. Psychological Reports, 112(2), 545-552. https://doi.org/10.2466/07.PR0.112.2.545-552

Levitt, M. J., Guacci-Franco, N., \& Levitt, J. L. (1993). Convoys of social support in childhood and early adolescence: Structure and function. Developmental Psychology, 29(5), 811-818. https://doi.org/10.1037/0012-1649.29.5.811

*Lima, M. L., Marques, S., Muiños, G., \& Camilo, C. (2017). All you need is Facebook friends? Associations between online and face-to-face friendships and health. Frontiers in Psychology, 8, Article 68.

https://doi.org/10.3389/fpsyg.2017.00068

Lipsey, M. W., \& Wilson, D. B. (2001). Practical meta-analysis. Sage.

Litwin, H., Stoeckel, K. J., \& Schwartz, E. (2015). Social networks and mental health among older Europeans: Are there age effects? European Journal of Ageing, 12(4), 299-309. https://doi.org/10.1007/s10433-015-0347-y

Liu, D., \& Baumeister, R. F. (2016). Social networking online and personality of self-worth: A meta-analysis. Journal of Research in Personality, 64, 79-89. https://doi.org/10.1016/j.jrp.2016.06.024

*Liu, J., Li, C., Carcioppolo, N., \& North, M. (2016). Do our Facebook friends make us feel worse? A study of social comparison and emotion. Human Communication Research, 42(4), 619-640. https://doi.org/10.1111/hcre.12090

*Locatelli, S. M., Kluwe, K., \& Bryant, F. B. (2012). Facebook use and the tendency to ruminate among college students: Testing mediational hypotheses. Journal of Educational Computing Research, 46(4), 377-394.

https://doi.org/10.2190/EC.46.4.d

Löckenhoff, C. E., \& Carstensen, L. L. (2004). Socioemotional selectivity theory, aging, and health: The increasingly delicate balance between regulating emotions and making tough choices. Journal of Personality, 72(6), 13951424. https://doi.org/10.1111/j.1467-6494.2004.00301.x

*Long, J. R. (2012). Relationship of self-concept, depression, academic estimation, and online social networking in adolescents [Unpublished master's professional paper]. University of Montana.

https://scholarworks.umt.edu/etd/814

*Lönnqvist, J.-E., \& Deters, F. G. (2016). Facebook friends, subjective well-being, social support, and personality. Computers in Human Behavior, 55(Part A), 113-120. https://doi.org/10.1016/j.chb.2015.09.002

*Lönnqvist, J.-E., \& Itkonen, J. V. A. (2014). It's all about extraversion: Why Facebook friend count doesn't count towards well-being. Journal of Research in Personality, 53, 64-67. https://doi.org/10.1016/j.jrp.2014.08.009 
*Manago, A. M., Taylor, T., \& Greenfield, P. M. (2012). Me and my 400 friends: The anatomy of college students' Facebook networks, their communication patterns, and well-being. Developmental Psychology, 48(2), 369-380. https://doi.org/10.1037/a0026338

*Marshall, T. C., Lefringhausen, K., \& Ferenczi, N. (2015). The Big Five, self-esteem, and narcissism as predictors of the topics people write about in Facebook status updates. Personality and Individual Differences, 85, 35-40. https://doi.org/10.1016/j.paid.2015.04.039

*Mazurek, M. O. (2013). Social media use among adults with autism spectrum disorders. Computers in Human Behavior, 29(4), 1709-1714. https://doi.org/10.1016/j.chb.2013.02.004

*Mersin, S., \& Acılar, A. (2015). Exploring the relationship between Facebook and self-esteem among Turkish university students. International Journal of Cyber Behavior, Psychology and Learning, 5(4), Article 5.

https://doi.org/10.4018/IJCBPL.2015100105

* Metzler, A., \& Scheithauer, H. (2017). The long-term benefits of positive self-presentation via profile pictures, number of friends and the initiation of relationships on Facebook for adolescents' self-esteem and the initiation of offline relationships. Frontiers in Psychology, 8, Article 1981. https://doi.org/10.3389/fpsyg.2017.01981

*Mikami, A. Y., Szwedo, D. E., Allen, J. P., Evans, M. A., \& Hare, A. L. (2010). Adolescent peer relationships and behavior problems predict young adults communication on social networking websites. Developmental Psychology, 46(1), 46-56. https://doi.org/10.1037/a0017420

*Moorman, J. (2012). How Facebook gave me 'friends': The impact of Facebook importance on engagement and psychological well-being [Master's thesis, Carleton University]. https://doi.org/10.22215/etd/2012-06858

Moreno, M. A., Jelenchick, L. A., Egan, K. G., Cox, E., Young, H., Gannon, K. E., \& Becker, T. (2011). Feeling bad on Facebook: Depression disclosures by college students on a social networking site. Depression and Anxiety, 28(6), 447-455. https://doi.org/10.1002/da.20805

*Morgan, J. (2010). The military and Facebook [Doctor Dissertation, Wright Institute Graduate School of Psychology].

*Morin-Major, J. K., Marin, M.-F., Durand, N., Wan, N., Juster, R.-P., \& Lupien, S. J. (2016). Facebook behaviors associated with diurnal cortisol in adolescents: Is befriending stressful? Psychoneuroendocrinology, 63, 238-246. https://doi.org/10.1016/j.psyneuen.2015.10.005

*Murphy, C. (2013). Facebook and Irish adolescents: The relationship between social media use, personality, and wellbeing [Master's thesis, Dublin Business School]. http://hdl.handle.net/10788/1584

*Nabi, R. L., Prestin, A., \& So, J. (2013). Facebook friends with (health) benefits? Exploring social network site use and perceptions of social support, stress, and well-being. Cyberpsychology, Behavior, and Social Networking, 16(10), 721-727. https://doi.org/10.1089/cyber.2012.0521

*Oh, H. J., Ozkaya, E., \& LaRose, R. (2014). How does online social networking enhance life satisfaction? The relationships among online supportive interaction, affect, perceived social support, sense of community, and life satisfaction. Computers in Human Behavior, 30, 69-78. https://doi.org/10.1016/j.chb.2013.07.053

*Ophir, Y., Asterhan, C. S. C., \& Schwarz, B. B. (2019). The digital footprints of adolescent depression, social rejection and victimization of bullying on Facebook. Computers in Human Behavior, 91, 62-71.

https://doi.org/10.1016/j.chb.2018.09.025

*Park, S., Kim, I., Lee, S. W., Yoo, J., Jeong, B., \& Cha, M. (2015). Manifestation of depression and loneliness on social networks: A case study of young adults on Facebook. In Proceedings of the 18th ACM Conference on 
Computer Supported Cooperative Work \& Social Computing (pp. 557-570). ACM.

https://doi.org/10.1145/2675133.2675139

*Park, S. Y., \& Baek, Y. M. (2018). Two faces of social comparison on Facebook: The interplay between social comparison orientation, emotions, and psychological well-being. Computers in Human Behavior, 79, 83-93. https://doi.org/10.1016/j.chb.2017.10.028

Perry, R., Drachen, A., Kearney, A., Kriglstein, S., Nacke, L. E., Sifa, R., Wallner, G., \& Johnson, D. (2018). Online-only friends, real-life friends or strangers? Differential associations with passion and social capital in video game play. Computers in Human Behavior, 79, 202-210. https://doi.org/10.1016/j.chb.2017.10.032

*Petrocchi, N., Asnaani, A., Martinez, A. P., Nadkarni, A., \& Hofmann, S. G. (2015). Differences between people who use only Facebook and those who use Facebook plus Twitter. International Journal of Human-Computer Interaction, 31(2), 157-165. https://doi.org/10.1080/10447318.2014.986640

*Phu, B., \& Gow, A. J. (2019). Facebook use and its association with subjective happiness and loneliness. Computers in Human Behavior, 92, 151-159. https://doi.org/10.1016/j.chb.2018.11.020

Pinquart, M., \& Sörensen, S. (2000). Influences of socioeconomic status, social network, and competence on subjective well-being in later life: A meta-analysis. Psychology and Aging, 15(2), 187-224.

https://doi.org/10.1037/0882-7974.15.2.187

Pollet, T. V., Roberts, S. G. B., \& Dunbar, R. I. M. (2011). Use of social network sites and instant messaging does not lead to increased offline social network size, or to emotionally closer relationships with offline network members. Cyberpsychology, Behavior, and Social Networking, 14(4), 253-258.

https://doi.org/10.1089/cyber.2010.0161

*Reinecke, L., \& Trepte, S. (2014). Authenticity and well-being on social network sites: A two-wave longitudinal study on the effects of online authenticity and the positivity bias in SNS communication. Computers in Human Behavior, 30, 95-102. https://doi.org/10.1016/j.chb.2013.07.030

Roberts, S. G. B., \& Dunbar, R. I. M. (2011). Communication in social networks: Effects of kinship, network size, and emotional closeness. Personal Relationships, 18(3), 439-452. https://doi.org/10.1111/j.1475-

$6811.2010 .01310 . x$

*Rogers, D. (2017). The negative effects of Facebook and social network sites [Master's thesis, Long Island University].

*Rosen, L. D., Whaling, K., Rab, S., Carrier, L. M., \& Cheever, N. A. (2013). Is Facebook creating "iDisorders"? The link between clinical symptoms of psychiatric disorders and technology use, attitudes and anxiety. Computers in Human Behavior, 29(3), 1243-1254. https://doi.org/10.1016/j.chb.2012.11.012

Rosenberg, M. (1965). Society and the adolescent self-image. Princeton University Press.

Rosenberg, M. S., Adams, D. C., \& Gurevitch, J. (2000). MetaWin: Statistical software for meta-analysis. Sinauer Associates.

Russell, D. W. (1996). UCLA Loneliness Scale (Version 3): Reliability, validity, and factor structure. Journal of Personality Assessment, 66(1), 20-40. https://doi.org/10.1207/s15327752.jpa6601_2

Ryff, C. D. (1989). Happiness is everything, or is it? Explorations on the meaning of psychological well-being. Journal of Personality and Social Psychology, 57(6), 1069-1081. https://doi.org/10.1037/0022-3514.57.6.1069 
Santini, Z. I., Koyanagi, A., Tyrovolas, S., Mason, C., \& Haro, J. M. (2015). The association between social relationships and depression: A systematic review. Journal of Affective Disorders. 175, 53-65.

https://doi.org/10.1016/j.jad.2014.12.049

*Schwartz, M. (2010). The usage of Facebook as it relates to narcissism, self-esteem and loneliness [Doctoral dissertation, Pace University]. https://digitalcommons.pace.edu/dissertations/AAI3415681

Seabrook, E. M., Kern. M. L., \& Rickard, N. S. (2016). Social networking sites, depression, and anxiety: A systematic review. JMIR Mental Health, 3(4), Article e50. https://doi.org/10.2196/mental.5842

*Seo, M., Kim, J., \& Yang, H. (2016). Frequent interaction and fast feedback predict perceived social support: Using crawled and self-reported data of Facebook users. Journal of Computer-Mediated Communication, 21(4), 282-297. https://doi.org/10.1111/jcc4.12160

*Seto, E. (2012). Associations between self-reported narcissism, self-esteem, and social-emotional functions of Facebook [Unpublished bachelor's thesis]. Baylor University. https://baylor-ir.tdl.org/handle/2104/8322

*Shchebetenko, S. (2019). Do personality characteristics explain the associations between self-esteem and online social networking behaviour? Computers in Human Behavior, 91, 17-23.

https://doi.org/10.1016/j.chb.2018.09.017

*Sheldon, P. (2012). Profiling the non-users: Examination of life-position indicators, sensation seeking, shyness, and loneliness among users and non-users of social network sites. Computers in Human Behavior, 28(5), 19601965. https://doi.org/10.1016/j.chb.2012.05.016

*Sherlock, M., \& Wagstaff, D. L. (2019). Exploring the relationship between frequency of Instagram use, exposure to idealized images, and psychological well-being in women. Psychology of Popular Media Culture, 8(4), 482-490. https://doi.org/10.1037/ppm0000182

*Singh, A. M. (2014). The relationship between Facebook use, loneliness, and self esteem among high school and undergraduate students [Master's thesis, Long Island University].

*Skues, J. L., Williams, B., \& Wise, L. (2012). The effects of personality traits, self-esteem, loneliness, and narcissism on Facebook use among university students. Computers in Human Behavior, 28(6), 2414-2419. https://doi.org/10.1016/j.chb.2012.07.012

Smith, K. P., \& Christakis, N. A. (2008). Social networks and health. Annual Review of Sociology, 34, 405-429. https://doi.org/10.1146/annurev.soc.34.040507.134601

Song, H., Zmyslinski-Seelig, A., Kim, J., Drent, A., Victor, A., Omori, K., \& Allen, M. (2014). Does Facebook make you lonely? A meta-analysis. Computers in Human Behavior, 36, 446-452. https://doi.org/10.1016/j.chb.2014.04.011

Statista. (2019). Distribution of Facebook users worldwide as of April 2019, by age and gender. https://www.statista.com/statistics/376128/facebook-global-user-age-distribution/

Suldo, S. M., \& Shaffer, E. J. (2008). Looking beyond psychopathology: The dual-factor model of mental health in youth. School Psychology Review. 37(1), 52-68. https://doi.org/10.1080/02796015.2008.12087908

*Tang, J. C. M., \& Livingston, M. G. (2012, June 16). Correlation between Facebook usage and loneliness and depression [Poster presentation]. The Hong Kong Psychological Society Annual Conference, Hong Kong. https://digitalcommons.csbsju.edu/psychology_pubs/71/

*Turel, O., \& Bechara, A. (2016). Social networking site use while driving: ADHD and the mediating roles of stress, self-esteem and craving. Frontiers in Psychology, 7, Article 455. https://doi.org/10.3389/fpsyg.2016.00455 
*Turel, O., \& Qahri-Saremi, H. (2016). Problematic use of social networking sites: Antecedents and consequence from a dual system theory perspective. Journal of Management Information Systems, 33(4), 1087-1116.

https://doi.org/10.1080/07421222.2016.1267529

*Utz, S., Tanis, M., \& Vermeulen, I. (2012). It is all about being popular: The effects of need for popularity on social network site use. Cyberpsychology, Behavior, and Social Networking, 15(1), 37-42.

https://doi.org/10.1089/cyber.2010.0651

Vahedi, Z., \& Zannella, L. (2019). The association between self-reported depressive symptoms and the use of social networking sites (SNS): A meta-analysis. Current Psychology. Advance online publication.

https://doi.org/10.1007/s12144-019-0150-6

Valentine, J. C. (2009). Judging the quality of primary research. In H. Cooper, L. V. Hedges, \& J. C. Valentine (Eds.), The handbook of research synthesis and meta-analysis (pp. 129-146). Russell Sage Foundation.

*Vanman, E. J., Baker, R., \& Tobin, S. J. (2018). The burden of online friends: the effects of giving up Facebook on stress and well-being. The Journal of Social Psychology, 158(4), 496-508.

https://doi.org/10.1080/00224545.2018.1453467

Wang, X. (2016). Subjective well-being associated with size of social network and social support of elderly. Journal of Health Psychology, 21(6), 1037-1042. https://doi.org/10.1177/1359105314544136

*Wang, Y., Nie, R., Li, Z., \& Zhou, N. (2018). WeChat Moments use and self-esteem among Chinese adults: The mediating roles of personal power and social acceptance and the moderating roles of gender and age.

Personality and Individual Differences, 131, 31-37. https://doi.org/10.1016/j.paid.2018.04.012

*Weidman, A. C., \& Levinson, C. A. (2015). I'm still socially anxious online: Offline relationship impairment characterizing social anxiety manifests and is accurately perceived in online social networking profiles.

Computers in Human Behavior, 49, 12-19. https://doi.org/10.1016/j.chb.2014.12.045

Westerhof, G., \& Keyes, C. (2010). Mental illness and mental health: The two continua model across the lifespan. Journal of Adult Development, 17(2), 110-119. https://doi.org/10.1007/s10804-009-9082-y

*Wilcox, K., \& Stephen, A. T. (2013). Are close friends the enemy? Online social networks, self-esteem, and selfcontrol. Journal of Consumer Research, 40(1), 90-103. https://doi.org/10.1086/668794

*Wright, K. B., Rosenberg, J., Egbert, N., Ploeger, N. A., Bernard, D. R., \& King, S. (2013). Communication competence, social support, and depression among college students: A model of Facebook and face-to-face support network influence. Journal of Health Communication, 18(1), 41-57.

https://doi.org/10.1080/10810730.2012.688250

Wrzus, C., Hänel, M., Wagner, J., \& Neyer, F. J. (2013). Social network changes and life events across the life span: A meta-analysis. Psychological Bulletin, 139(1), 53-80. https://doi.org/10.1037/a0028601

*Yang, C.-c. (2016). Instagram use, loneliness, and social comparison orientation: Interact and browse on social media, but don't compare. Cyberpsychology, Behavior, and Social Networking, 19(12), 703-708.

https://doi.org/10.1089/cyber.2016.0201

*Yang, C.-C., \& Brown, B. (2016). Online self-presentation on Facebook and self development during the college transition. Journal of Youth and Adolescence, 45(2), 402-416. https://doi.org/10.1007/s10964-015-0385-y

*Yoo, J. H., \& Jeong, E. J. (2017). Psychosocial effects of SNS use: A longitudinal study focused on the moderation effect of social capital. Computers in Human Behavior, 69, 108-119. https://doi.org/10.1016/j.chb.2016.12.011 
Yoon, S., Kleinman, M., Mertz, J., \& Brannick, M. (2019). Is social network site usage related to depression? A meta-analysis of Facebook-depression relations. Journal of Affective Disorders, 248, 65-72.

https://doi.org/10.1016/j.jad.2019.01.026

*Young, J. M. (2015). College students' friendships, self-esteem, and experience using Facebook [Unpublished master's thesis]. Middle Tennessee State University.

*Zabawska, M. (2013). Like it or not. The relationship between personality traits, narcissism, self-esteem, selfmonitoring and Facebook usage [Unpublished bachelor's thesis]. Dublin Business School.

*Zeeni, N., Doumit, R., Kharma, J. A., \& Sanchez-Ruiz, M.-J. (2018). Media, technology use, and attitudes: Associations with physical and mental well-being in youth with implications for evidence-based practice. Worldviews on Evidence Based Nursing, 15(4), 304-312. https://doi.org/10.1111/wvn.12298

*Zell, A. L., \& Moeller, L. (2018). Are you happy for me ... on Facebook? The potential importance of "likes" and comments. Computers in Human Behavior, 78, 26-33. https://doi.org/10.1016/j.chb.2017.08.050

*Zhang, R. (2017). The stress-buffering effect of self-disclosure on Facebook: An examination of stressful life events, social support, and mental health among college students. Computers in Human Behavior, 75, 527-537. https://doi.org/10.1016/j.chb.2017.05.043

\section{Correspondence to:}

Chiungjung Huang

Graduate Institute of Education

National Changhua University of Education

1 Jinde Road

Changhua, 500

Taiwan

Email: chiung(at)cc.ncue.edu.tw

Editorial record: First submission received on September 23, 2020. Revision received on February 12, 2021. Accepted for publication on March 17, 2021.

Editor in charge: David Smahel

\section{About Authors}

Chiungjung Huang, Ph.D., is a Professor at National Changhua University of Education, Graduate Institute of Education. Her research focuses on meta-analysis and social media use. 